\title{
Gait analysis of national athletes after anterior cruciate ligament reconstruction following three stages of rehabilitation program: symmetrical perspective
}

\begin{abstract}
This study aimed to objectively evaluate changes in gait kinematics, kinetics and symmetry among anterior cruciate ligament (ACL) reconstructed athletes during rehabilitation. Twentytwo national athletes with ACL reconstruction and 15 healthy athletes were recruited for the study. Gait data were collected between the weeks 4-5, 8-9, and 12-13 post-operation using three-dimensional motion analysis system. Five separate components, including knee range of motion (ROM), vertical ground reaction force (VGRF), their symmetries and knee extension moment were evaluated. One way and repeated measure multivariate analysis of variance (MANOVA) were used to analyze the knee ROMs. The VGRF and extension moment were tested using repeated measure ANOVA and independent sample $t$-test. Findings indicated significant alterations in all measured components between patients' Test 1 and control group. Repeated measure analysis revealed significant effect for time in components of knee angular and $\operatorname{VGRF}(P<0.001)$, their symmetry index $(P=0.03)$ and knee extension moment $(P=0.045)$. Univariate outcomes demonstrated significant improvement in the injured limb's stance and swing $(P<0.001)$, and single-stance $(P=0.005)$ ROMs over time. Symmetry indexes of stance and swing ROM, and VGRF reduced significantly by $26.3 \%(P=0.001), 17.9 \% \quad(P<0.001)$, and $31.9 \% \quad(P=0.03)$ respectively. After three months, symmetry indexes of single-stance ROM and VGRF along with operated knee extension moment were the only variables which showed significant differences with control group. The rehabilitation program allowed national athletes to restore the operated limb's gait parameters except knee extension moment by $12-13$ weeks postreconstruction; however, more time is required to normalize single-stance ROM and VGRF asymmetries.
\end{abstract}

Keyword: Gait analysis; Symmetry; Anterior cruciate ligament reconstruction; Knee range of motion; Ground reaction force; Knee extension moment 